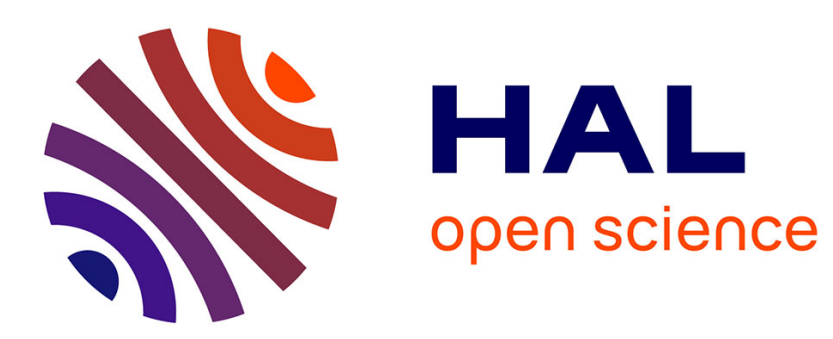

\title{
Microscopic local conservation laws for classical fluids
}

Roger Balian

\section{To cite this version:}

Roger Balian. Microscopic local conservation laws for classical fluids. The European Physical Journal. Special Topics, 2015, 224, pp.927-934. 10.1140/epjst/e2015-02436-5 . cea-01114504

\section{HAL Id: cea-01114504 https://hal-cea.archives-ouvertes.fr/cea-01114504}

Submitted on 9 Feb 2015

HAL is a multi-disciplinary open access archive for the deposit and dissemination of scientific research documents, whether they are published or not. The documents may come from teaching and research institutions in France or abroad, or from public or private research centers.
L'archive ouverte pluridisciplinaire HAL, est destinée au dépôt et à la diffusion de documents scientifiques de niveau recherche, publiés ou non, émanant des établissements d'enseignement et de recherche français ou étrangers, des laboratoires publics ou privés. 


\title{
Microscopic local conservation laws for classical fluids
}

\author{
Roger Balian \\ Institut de Physique Théorique, Centre de Saclay, F-91191 Gif sur Yvette Cx.
}

\begin{abstract}
The local densities and current densities of conserved quantities are expressed, for a fluid under arbitrary off-equilibrium conditions, in terms of the two-particle potential $W(r)$ and of the one- and two-particle densities in phase space $f$ and $f_{2}$. When $f$ and $f_{2}$ vary significantly over the range of $W(r)$, the density and current density of energy are not defined in a unique fashion, so that conservation of energy can be implemented locally in many different ways. Owing to Galilean invariance, the stress tensor and the heat flux are defined even far from the hydrodynamic regime.
\end{abstract}

In the 1960's, Jacques Yvon was professor of physics at the University of Paris. Beforehand, he had directed the Department of physics and nuclear reactors at Saclay. He therefore asked Cirano De Dominicis, then myself, who worked in this department, to become his assistant for his course of statistical mechanics. He focused on classical fluids, modelled as an assembly of $N$ point particles with mass $m$ interacting through a two-body potential $W\left(\left|\mathbf{r}_{j}-\mathbf{r}_{k}\right|\right)$. His lectures started from the most fundamental dynamical description, based on the Liouville equation governing the density in the $6 N$-dimensional phase space, a function of the time-dependent coordinates $\mathbf{r}_{j}$ and $\mathbf{p}_{j}$ $(1 \leq j \leq N)$. Having defined the reduced 1-body, 2-body, ... densities

$$
\begin{gathered}
f(\mathbf{r}, \mathbf{p} ; t) \equiv\left\langle\sum_{j} \delta^{3}\left(\mathbf{r}_{j}-\mathbf{r}\right) \delta^{3}\left(\mathbf{p}_{j}-\mathbf{p}\right)\right\rangle \\
f_{2}\left(\mathbf{r}, \mathbf{p} ; \mathbf{r}^{\prime}, \mathbf{p}^{\prime} ; t\right) \equiv\left\langle\sum_{j \neq k} \delta^{3}\left(\mathbf{r}_{j}-\mathbf{r}\right) \delta^{3}\left(\mathbf{p}_{j}-\mathbf{p}\right) \delta^{3}\left(\mathbf{r}_{k}-\mathbf{r}^{\prime}\right) \delta^{3}\left(\mathbf{p}_{k}-\mathbf{p}^{\prime}\right)\right\rangle, \ldots,
\end{gathered}
$$

where $\langle\ldots\rangle$ is the expectation value over positions and momenta in phase space, he derived the BBGKY hierarchy of equations that he had initiated long ago [1]. Among the many topics he treated thereafter, he established rigorous foundations for conservation laws in classical statistical mechanics; this part of his course inspires the present discussion.

From the global conservation of the particle number $N$, of the energy $E$, of the three components of the momentum $\mathbf{P}$ and of the angular momentum $\mathbf{L}$, we infer the existence at each point $\mathbf{r}$ of densities $\rho_{N}$ of particles, $\rho_{E}$ of energy, $\rho_{\mathbf{P}}$ of momentum and $\rho_{\mathbf{L}}$ of angular momentum, and the existence of corresponding current densities $\mathbf{J}_{N}, \mathbf{J}_{E}, \mathbf{J}_{\mathbf{P}}$ and $\mathbf{J}_{\mathbf{L}}$ that satisfy local conservation laws $\partial \rho / \partial t+\operatorname{div} \mathbf{J}=0$. Our 
purpose [2] is to define these quantities without approximations, for an arbitrary potential $W$ having possibly a long range, and in any regime even far from local equilibrium, for instance within shock waves or near boundaries.

The density of particles is

$$
\rho_{N}(\mathbf{r}, t)=\left\langle\sum_{j} \delta^{3}\left(\mathbf{r}_{j}-\mathbf{r}\right)\right\rangle=\int \mathrm{d}^{3} \mathbf{p} f(\mathbf{r}, \mathbf{p} ; t) .
$$

Its time derivative follows from Hamilton's equations $\mathrm{d} \mathbf{r}_{j} / \mathrm{d} t=\mathbf{v}_{j}=\mathbf{p}_{j} / m, \mathrm{~d} \mathbf{p}_{j} / \mathrm{d} t=$ $\mathbf{F}_{j}$, so that the conservation of particle number $\partial \rho_{N} / \partial t+\operatorname{div} \mathbf{J}_{N}=0$ is readily expressed in terms of the current density of particles

$$
\mathbf{J}_{N}(\mathbf{r}, t)=\left\langle\sum_{j} \mathbf{v}_{j} \delta^{3}\left(\mathbf{r}_{j}-\mathbf{r}\right)\right\rangle=\int \mathrm{d}^{3} \mathbf{p} \frac{\mathbf{p}}{m} f(\mathbf{r}, \mathbf{p} ; t) .
$$

As regards the conservation of momentum, after having defined the three components of the density of momentum through

$$
\rho_{\mathbf{P}}(\mathbf{r}, t)=\left\langle\sum_{j} \mathbf{p}_{j} \delta^{3}\left(\mathbf{r}_{j}-\mathbf{r}\right)\right\rangle=\int \mathrm{d}^{3} \mathbf{p} \mathbf{p} f(\mathbf{r}, \mathbf{p} ; t),
$$

we need to express their time derivative (involving the velocities $\mathbf{v}_{j}$ and the forces $\mathbf{F}_{j}$ issued from the potential $W$ ),

$$
\frac{\partial \rho_{\mathbf{P} \beta}}{\partial \mathbf{t}}(\mathbf{r}, t)=\left\langle\sum_{j, \alpha} v_{j \alpha} p_{\beta} \frac{\partial \delta^{3}\left(\mathbf{r}_{j}-\mathbf{r}\right)}{\partial r_{j \alpha}}\right\rangle-\left\langle\sum_{j \neq k} \frac{\partial W\left(\left|\mathbf{r}_{j}-\mathbf{r}_{k}\right|\right)}{\partial r_{j \beta}} \delta^{3}\left(\mathbf{r}_{j}-\mathbf{r}\right)\right\rangle,
$$

in the form of the divergence $-\sum_{\alpha} \partial \mathbf{J}_{\mathbf{P} \beta}^{\alpha} / \partial r_{\alpha}$ of a tensor $\mathbf{J}_{\mathbf{P} \beta}^{\alpha}(\mathbf{r}, t)$. This is straightforward for the first term. We rewrite the second term as

$$
-\frac{1}{2} \int \mathrm{d}^{3} \mathbf{r}^{\prime} \frac{r_{\beta}^{\prime}}{r^{\prime}} \frac{\mathrm{d} W\left(r^{\prime}\right)}{\mathrm{d} r^{\prime}}\left\langle\sum_{j \neq k} \delta^{3}\left(\mathbf{r}^{\prime}-\mathbf{r}_{j}+\mathbf{r}_{k}\right)\left[\delta^{3}\left(\mathbf{r}_{j}-\mathbf{r}\right)-\delta^{3}\left(\mathbf{r}_{k}-\mathbf{r}\right)\right]\right\rangle,
$$

and transform the last bracket into a divergence according to

$$
\begin{aligned}
& \delta^{3}\left(\mathbf{r}_{j}-\mathbf{r}\right)-\delta^{3}\left(\mathbf{r}_{k}-\mathbf{r}\right)=\int_{-1}^{1} \mathrm{~d} \lambda \frac{\mathrm{d} \delta^{3}\left[\mathbf{r}-\frac{1}{2}(1+\lambda) \mathbf{r}_{j}-\frac{1}{2}(1-\lambda) \mathbf{r}_{k}\right]}{\mathrm{d} \lambda} \\
& =-\frac{1}{2} \sum_{\alpha} \frac{\partial}{\partial r_{\alpha}} \int_{-1}^{1} \mathrm{~d} \lambda\left(r_{j \alpha}-r_{k \alpha}\right) \delta^{3}\left[\mathbf{r}-\frac{1}{2}(1+\lambda) \mathbf{r}_{j}-\frac{1}{2}(1-\lambda) \mathbf{r}_{k}\right] .
\end{aligned}
$$

This finally yields the expression of the conserved current density of momentum:

$$
\begin{gathered}
\mathbf{J}_{\mathbf{P} \beta}^{\alpha}(\mathbf{r}, t)=\int \mathrm{d}^{3} \mathbf{p} \frac{p_{\alpha} p_{\beta}}{m} f(\mathbf{r}, \mathbf{p} ; t)-\frac{1}{4} \int \mathrm{d}^{3} \mathbf{p} \mathrm{d}^{3} \mathbf{p}^{\prime} \mathrm{d}^{3} \mathbf{r}^{\prime} \\
\times \frac{r_{\alpha}^{\prime} r_{\beta}^{\prime}}{r^{\prime}} \frac{\mathrm{d} W\left(r^{\prime}\right)}{\mathrm{d} r^{\prime}} \int_{-1}^{1} \mathrm{~d} \lambda f_{2}\left[\mathbf{r}+\frac{1}{2}(1-\lambda) \mathbf{r}^{\prime}, \mathbf{p} ; \mathbf{r}-\frac{1}{2}(1+\lambda) \mathbf{r}^{\prime}, \mathbf{p}^{\prime} ; t\right] .
\end{gathered}
$$


Due to the non-locality of the potential, $\mathbf{J}_{\mathbf{P}}(\mathbf{r}, t)$ involves contributions from the twobody function $f_{2}$ taken at points located on each side of $\mathbf{r}$, at distances of the order of the range of $W$.

The above results for the local balance of momentum can alternatively be derived through Noether's approach. The conservation of the total momentum results from the invariance of the Lagrangian under the translation $\mathbf{r}_{j} \mapsto \mathbf{r}_{j}+\delta \mathbf{r}_{j}$ by a small vector $\delta \mathbf{r}_{j}=\mathbf{a}$. Consider now a slowly varying infinitesimal vector field $\mathbf{a}(\mathbf{r}, t)$. Given a solution $\left\{\mathbf{r}_{j}(t)\right\}$ of the equations of motion in the $3 N$-dimensional space, a change of $\mathbf{r}_{j}(t)$ into $\mathbf{r}_{j}(t)+\mathbf{a}\left[\mathbf{r}_{j}(t), t\right]$ produces a variation of the action around this solution, which has the form

$$
\delta S=\int \mathrm{d}^{3} \mathbf{r} \mathrm{d} t\left[\sum_{\beta} \frac{\partial a_{\beta}(\mathbf{r}, t)}{\partial t} \rho_{\mathbf{P} \beta}(\mathbf{r}, t)+\sum_{\alpha, \beta} \frac{\partial a_{\beta}(\mathbf{r}, t)}{\partial r_{\alpha}} \mathbf{J}_{\mathbf{P} \beta}^{\alpha}(\mathbf{r}, t)\right]
$$

in terms of the time derivative and of the gradient of $\mathbf{a}(\mathbf{r}, t)$. The coefficients $\rho_{\mathbf{P} \beta}(\mathbf{r}, t)$ and $\mathbf{J}_{\mathbf{P} \beta}^{\alpha}(\mathbf{r}, t)$ arising from this calculation are found to be equal to (5) and (8), respectively. Integration by parts yields the local conservation law of momentum, owing to the stationarity of $S$ for arbitrary $\mathbf{a}(\mathbf{r}, t)$.

In the above derivation of the momentum balance at the point $\mathbf{r}$, after having written in (6) the force exerted by the particle $k$ onto the particle $j$ in terms of the potential $W\left(\left|\mathbf{r}_{j}-\mathbf{r}_{k}\right|\right)$, we have transferred through Eq. (7) this force to the running points $\mathbf{r}$ located between $\mathbf{r}_{j}$ and $\mathbf{r}_{k}$. Likewise, the contribution of the potential $W\left(\mid \mathbf{r}_{j}-\right.$ $\left.\mathbf{r}_{k} \mid\right)$ to the energy density $\rho_{E}(\mathbf{r}, t)$ should be transferred to $\mathbf{r}$. We are thus led to distribute the potential energy $W\left(\left|\mathbf{r}_{j}-\mathbf{r}_{k}\right|\right)$ over the segment $\left(\mathbf{r}_{j}, \mathbf{r}_{k}\right)$. Characterising as above a point $\mathbf{r}=\frac{1}{2}(1+\lambda) \mathbf{r}_{j}+\frac{1}{2}(1-\lambda) \mathbf{r}_{k}$ on $\left(\mathbf{r}_{j}, \mathbf{r}_{k}\right)$ by the parameter $\lambda$, we introduce on the segment $-1 \leq \lambda \leq 1$ a normalised measure $\mathrm{d} \chi / \mathrm{d} \lambda$, generated by a function $\chi(\lambda)$ that increases from $\chi(-1)=-\frac{1}{2}$ to $\chi(1)=\frac{1}{2}$. The density of energy is then defined as

$$
\begin{aligned}
\rho_{E}(\mathbf{r}, t) & =\left\langle\sum_{j} \frac{p_{j}^{2}}{2 m} \delta^{3}\left(\mathbf{r}_{j}-\mathbf{r}\right)\right\rangle \\
& +\left\langle\frac{1}{2} \sum_{j \neq k} \int_{-1}^{1} \mathrm{~d} \lambda \frac{\mathrm{d} \chi}{\mathrm{d} \lambda} W\left(\left|\mathbf{r}_{j}-\mathbf{r}_{k}\right|\right) \delta^{3}\left[\mathbf{r}-\frac{1}{2}(1+\lambda) \mathbf{r}_{j}-\frac{1}{2}(1-\lambda) \mathbf{r}_{k}\right]\right\rangle \\
& =\int \mathrm{d}^{3} \mathbf{p} \frac{p^{2}}{2 m} f(\mathbf{r}, \mathbf{p} ; t)+\frac{1}{2} \int \mathrm{d}^{3} \mathbf{r}^{\prime} \mathrm{d}^{3} \mathbf{p} \mathrm{d}^{3} \mathbf{p}^{\prime} \\
& \times W\left(r^{\prime}\right) \int_{-1}^{1} \mathrm{~d} \lambda \frac{\mathrm{d} \chi}{d \lambda} f_{2}\left[\mathbf{r}+\frac{1}{2}(1-\lambda) \mathbf{r}^{\prime}, \mathbf{p} ; \mathbf{r}-\frac{1}{2}(1+\lambda) \mathbf{r}^{\prime}, \mathbf{p}^{\prime} ; t\right] .
\end{aligned}
$$

Its time derivative is given by 


$$
\begin{gathered}
\frac{\partial \rho_{E}(\mathbf{r}, t)}{\partial t}=-\left\langle\sum_{j \neq k, \beta} v_{j \beta} \frac{\partial W\left(\left|\mathbf{r}_{j}-\mathbf{r}_{k}\right|\right)}{\partial r_{j \beta}} \delta^{3}\left(\mathbf{r}_{j}-\mathbf{r}\right)\right\rangle+\left\langle\sum_{j, \alpha} \frac{p_{j}^{2}}{2 m} v_{j \alpha} \frac{\partial \delta^{3}\left(\mathbf{r}_{j}-\mathbf{r}\right)}{\partial r_{j \alpha}}\right\rangle \\
-\left\langle\frac{1}{2} \sum_{j \neq k, \alpha} \int_{-1}^{1} \mathrm{~d} \lambda \frac{\mathrm{d} \chi}{\mathrm{d} \lambda} W\left(\left|\mathbf{r}_{j}-\mathbf{r}_{k}\right|\right)\left[\frac{1}{2}(1+\lambda) v_{j \alpha}+\frac{1}{2}(1-\lambda) v_{k \alpha}\right]\right. \\
\left.\times \frac{\partial \delta^{3}\left[\mathbf{r}-\frac{1}{2}(1+\lambda) \mathbf{r}_{j}-\frac{1}{2}(1-\lambda) \mathbf{r}_{k}\right]}{\partial r_{\alpha}}\right\rangle \\
+\left\langle\sum_{j \neq k, \beta} \int_{-1}^{1} \mathrm{~d} \lambda \frac{\mathrm{d} \chi}{\mathrm{d} \lambda}\left(v_{j \beta}-v_{k \beta}\right) \frac{\partial W\left(\left|\mathbf{r}_{j}-\mathbf{r}_{k}\right|\right)}{2 \partial r_{j \beta}} \delta^{3}\left[\mathbf{r}-\frac{1}{2}(1+\lambda) \mathbf{r}_{j}-\frac{1}{2}(1-\lambda) \mathbf{r}_{k}\right]\right\rangle
\end{gathered}
$$

While the two middle terms readily produce a divergence with respect to $\mathbf{r}$, the first and last terms should be combined so as to account for exchanges between kinetic and potential energies. Symmetrizing the first one with respect to $j$ and $k$, integrating by parts over $\lambda$, then using (7), we find their sum as

$$
\begin{aligned}
& \frac{1}{2} \int \mathrm{d}^{3} \mathbf{r}^{\prime}\left\langle\sum_{j \neq k, \beta} \frac{r_{\beta}^{\prime}}{r^{\prime}} \frac{\mathrm{d} W\left(r^{\prime}\right)}{\mathrm{d} r^{\prime}} \delta^{3}\left(\mathbf{r}^{\prime}-\mathbf{r}_{j}+\mathbf{r}_{k}\right) \int_{-1}^{1} \mathrm{~d} \lambda \frac{\mathrm{d} \chi}{\mathrm{d} \lambda}\right. \\
& \times\left\{\left[\delta^{3}\left[\mathbf{r}-\frac{1}{2}(1+\lambda) \mathbf{r}_{j}-\frac{1}{2}(1-\lambda) \mathbf{r}_{k}\right]-\delta^{3}\left(\mathbf{r}-\mathbf{r}_{j}\right)\right] v_{j \beta}\right. \\
& \left.\left.-\left[\delta^{3}\left[\mathbf{r}-\frac{1}{2}(1+\lambda) \mathbf{r}_{j}-\frac{1}{2}(1-\lambda) \mathbf{r}_{k}\right]-\delta^{3}\left(\mathbf{r}-\mathbf{r}_{k}\right)\right] v_{k \beta}\right\}\right\rangle \\
& =\frac{1}{4} \int \mathrm{d}^{3} \mathbf{r}^{\prime}\left\langle\sum_{j \neq k, \alpha, \beta} \frac{r_{\beta}^{\prime}}{r^{\prime}} \frac{\mathrm{d} W\left(r^{\prime}\right)}{\mathrm{d} r^{\prime}} \delta^{3}\left(\mathbf{r}^{\prime}-\mathbf{r}_{j}+\mathbf{r}_{k}\right) \int_{-1}^{1} \mathrm{~d} \lambda\right. \\
& \left.\times\left\{\left[\frac{1}{2}+\chi(\lambda)\right] v_{j \beta}+\left[\frac{1}{2}-\chi(\lambda)\right] v_{k \beta}\right\} r_{\alpha}^{\prime} \frac{\partial \delta^{3}\left[\mathbf{r}-\frac{1}{2}(1+\lambda) \mathbf{r}_{j}-\frac{1}{2}(1-\lambda) \mathbf{r}_{k}\right]}{\partial r_{\alpha}}\right\rangle .
\end{aligned}
$$

Altogether, the current density of energy is found as

$$
\begin{gathered}
\mathbf{J}_{E}(\mathbf{r}, t)=\int \mathrm{d}^{3} \mathbf{p} \frac{\mathbf{p}}{m} \frac{p^{2}}{2 m} f(\mathbf{r}, \mathbf{p} ; t)+\frac{1}{2 m} \int \mathrm{d}^{3} \mathbf{r}^{\prime} \mathrm{d}^{3} \mathbf{p} \mathrm{d}^{3} \mathbf{p}^{\prime} \int_{-1}^{1} \mathrm{~d} \lambda \\
\times\left\{W\left(r^{\prime}\right) \frac{\mathrm{d} \chi}{\mathrm{d} \lambda}\left[\frac{(1+\lambda)}{2} \mathbf{p}+\frac{(1-\lambda)}{2} \mathbf{p}^{\prime}\right]-\mathbf{r}^{\prime} \sum_{\beta} \frac{r_{\beta}^{\prime}}{r^{\prime}} \frac{\mathrm{d} W\left(r^{\prime}\right)}{2 \mathrm{~d} r^{\prime}}\left[\left(\frac{1}{2}+\chi\right) p_{\beta}+\left(\frac{1}{2}-\chi\right) p_{\beta}^{\prime}\right]\right\} \\
\times f_{2}\left[\mathbf{r}+\frac{1}{2}(1-\lambda) \mathbf{r}^{\prime}, \mathbf{p} ; \mathbf{r}-\frac{1}{2}(1+\lambda) \mathbf{r}^{\prime}, \mathbf{p}^{\prime} ; t\right]
\end{gathered}
$$

The conservation of energy can therefore be locally implemented in many different ways. This arbitrariness depends on the choice of the function $\chi(\lambda)$ that enters the above definitions of the density and flux of energy. If we take $\mathrm{d} \chi / \mathrm{d} \lambda=\delta(\lambda)$, the contribution $W\left(\left|\mathbf{r}_{j}-\mathbf{r}_{k}\right|\right)$ to the potential energy is assigned to the middle of the segment $\left(\mathbf{r}_{j}, \mathbf{r}_{k}\right)$; if $\mathrm{d} \chi / \mathrm{d} \lambda=\frac{1}{2}[\delta(\lambda+1)+\delta(\lambda-1)]$, it is assigned in half to $\mathbf{r}_{j}$ and $\mathbf{r}_{k}$; if $\chi(\lambda)=\lambda / 2$, it is uniformly spread over $\left(\mathbf{r}_{j}, \mathbf{r}_{k}\right)$. This choice of $\chi(\lambda)$ is irrelevant if the factor $f_{2}$ entering $(8),(10)$ and (11) does not vary significantly when 
$\mathbf{r}$ is translated over a distance smaller than the range of the potential $W$; in this case, these expressions simplify into

$$
\begin{aligned}
\mathbf{J}_{\mathbf{P} \beta}^{\alpha}(\mathbf{r}, t)= & \int \mathrm{d}^{3} \mathbf{p} \frac{p_{\alpha} p_{\beta}}{m} f(\mathbf{r}, \mathbf{p} ; t) \\
- & \frac{1}{4} \int \mathrm{d}^{3} \mathbf{p} \mathrm{d}^{3} \mathbf{p}^{\prime} \mathrm{d}^{3} \mathbf{r}^{\prime} \frac{r_{\alpha}^{\prime} r_{\beta}^{\prime}}{r^{\prime}} \frac{\mathrm{d} W\left(r^{\prime}\right)}{\mathrm{d} r^{\prime}} f_{2}\left(\mathbf{r}+\frac{1}{2} \mathbf{r}^{\prime}, \mathbf{p} ; \mathbf{r}-\frac{1}{2} \mathbf{r}^{\prime}, \mathbf{p}^{\prime} ; t\right), \\
\rho_{E}(\mathbf{r}, t)= & \int \mathrm{d}^{3} \mathbf{p} \frac{p^{2}}{2 m} f(\mathbf{r}, \mathbf{p} ; t) \\
+ & \frac{1}{2} \int \mathrm{d}^{3} \mathbf{r}^{\prime} \mathrm{d}^{3} \mathbf{p} \mathrm{d}^{3} \mathbf{p}^{\prime} W\left(r^{\prime}\right) f_{2}\left(\mathbf{r}+\frac{1}{2} \mathbf{r}^{\prime}, \mathbf{p} ; \mathbf{r}-\frac{1}{2} \mathbf{r}^{\prime}, \mathbf{p}^{\prime} ; t\right), \\
\mathbf{J}_{E}(\mathbf{r}, t) & =\int \mathrm{d}^{3} \mathbf{p} \frac{\mathbf{p}}{m} \frac{p^{2}}{2 m} f(\mathbf{r}, \mathbf{p} ; t)+\frac{1}{4 m} \int \mathrm{d}^{3} \mathbf{r}^{\prime} \mathrm{d}^{3} \mathbf{p} \mathrm{d}^{3} \mathbf{p}^{\prime} \\
& \times\left[W\left(r^{\prime}\right)\left(\mathbf{p}+\mathbf{p}^{\prime}\right)-\mathbf{r}^{\prime} \sum_{\beta} \frac{r_{\beta}^{\prime}}{r^{\prime}} \frac{\mathrm{d} W\left(r^{\prime}\right)}{d r^{\prime}}\left(p_{\beta}+p_{\beta}^{\prime}\right)\right] \\
& \times f_{2}\left(\mathbf{r}+\frac{1}{2} \mathbf{r}^{\prime}, \mathbf{p} ; \mathbf{r}-\frac{1}{2} \mathbf{r}^{\prime}, \mathbf{p}^{\prime} ; t\right) .
\end{aligned}
$$

However, either for long-range forces or in situations involving rapid space variations, the various formulations of the energy conservation differ and one should retain the expressions (8), (10) and (11).

The conservation of the total angular momentum $\mathbf{L}$ is related to the invariance of the Lagrangian under a rotation $\delta \mathbf{r}_{j}(t)=\boldsymbol{\omega} \wedge \mathbf{r}_{j}(t)$. Here again, one can use Noether's method, replacing the rotation vector $\boldsymbol{\omega}$ by an infinitesimal vector field $\boldsymbol{\omega}(\mathbf{r}, t)$, and evaluating the variation $\delta S$ of the action when the coordinates $\mathbf{r}_{j}(t)$ are transformed into $\mathbf{r}_{j}(t)+\boldsymbol{\omega}\left[\mathbf{r}_{j}(t), t\right] \wedge \mathbf{r}_{j}(t)$. The coefficients yield as in (9) the components of the local density $\rho_{\mathbf{L}}(\mathbf{r}, t)$ and current density $\mathbf{J}_{\mathbf{L}}(\mathbf{r}, t)$ of angular momentum, the stationarity of the action implying the local conservation of angular momentum $\partial \rho_{\mathbf{L}} / \partial t+\operatorname{div} \mathbf{J}_{\mathbf{L}}=0$. In fact, the calculation is the same as in (9) within replacement of $\mathbf{a}\left[\mathbf{r}_{j}(t), t\right]$ by $\boldsymbol{\omega}\left[\mathbf{r}_{j}(t), t\right] \wedge \mathbf{r}_{j}(t)$. Without even writing the specific forms of $\rho_{\mathbf{P}}, \rho_{\mathbf{L}}, \mathbf{J}_{\mathbf{P}}$ and $\mathbf{J}_{\mathbf{L}}$, one finds

$$
\rho_{\mathbf{L}}(\mathbf{r}, t)=\mathbf{r} \wedge \rho_{\mathbf{P}}(\mathbf{r}, t), \quad \mathbf{J}_{\mathbf{L}}^{\alpha}(\mathbf{r}, t)=\mathbf{r} \wedge \mathbf{J}_{\mathbf{P}}^{\alpha}(\mathbf{r}, t),
$$

relations valid for point particles without intrinsic angular momentum. Together with (15), the local conservation of angular momentum entails the symmetry of the tensor $\mathbf{J}_{\mathbf{P} \beta}^{\alpha}(\mathbf{r}, t)$. The latter property, readily checked on (8), is thus a consequence of rotational invariance.

We now consider the consequences of Galilean invariance. If the frame is set into motion with a velocity $\mathbf{u}$, the coordinates $\mathbf{r}, \mathbf{p}$ are changed into $\mathbf{r}^{\prime}=\mathbf{r}-\mathbf{u}$, $\mathbf{p}^{\prime}=\mathbf{p}-m \mathbf{u}$, the total momentum into $\mathbf{P}^{\prime}=\mathbf{P}-m \mathbf{u} N$ and the energy into $E^{\prime}=$ $E-\mathbf{u} \cdot \mathbf{P}+\frac{1}{2} m u^{2} N$. Accordingly, the density of momentum acquires a contribution from the density of particles, and the density of energy acquires contributions from the densities of momentum and particles, according to

$$
\begin{aligned}
& \rho_{N}^{\prime}\left(\mathbf{r}^{\prime}, t\right)=\rho_{N}(\mathbf{r}, t), \quad \rho_{\mathbf{P}^{\prime}}^{\prime}\left(\mathbf{r}^{\prime}, t\right)=\rho_{\mathbf{P}}(\mathbf{r}, t)-m \mathbf{u} \rho_{N}(\mathbf{r}, t) \\
& \rho_{E^{\prime}}^{\prime}\left(\mathbf{r}^{\prime}, t\right)=\rho_{E}(\mathbf{r}, t)-\sum_{\beta} u_{\beta} \rho_{\mathbf{P} \beta}(\mathbf{r}, t)+\frac{1}{2} m u^{2} \rho_{N}(\mathbf{r}, t)
\end{aligned}
$$


Besides the change of $\mathbf{P}$ into $\mathbf{P}^{\prime}$ and $E$ into $E^{\prime}$, the transformation of the current densities should account for the motion of the frame, so as to ensure that the equations of conservation hold in both frames. The conserved currents in the moving frame are therefore

$$
\begin{aligned}
\mathbf{J}_{N}^{\prime}\left(\mathbf{r}^{\prime}, t\right) & =\mathbf{J}_{N}(\mathbf{r}, t)-\mathbf{u} \rho_{N}^{\prime}\left(\mathbf{r}^{\prime}, t\right), \\
\mathbf{J}_{\mathbf{P}^{\prime} \beta}^{\prime}\left(\mathbf{r}^{\prime}, t\right) & =\mathbf{J}_{\mathbf{P} \beta}(\mathbf{r}, t)-m u_{\beta} \mathbf{J}_{N}(\mathbf{r}, t)-\mathbf{u} \rho_{\mathbf{P}^{\prime} \beta}^{\prime}\left(\mathbf{r}^{\prime}, t\right), \\
\mathbf{J}_{E^{\prime}}^{\prime}\left(\mathbf{r}^{\prime}, t\right) & =\mathbf{J}_{E}(\mathbf{r}, t)-\sum_{\beta} u_{\beta} \mathbf{J}_{\mathbf{P} \beta}(\mathbf{r}, t)+\frac{1}{2} m u^{2} \mathbf{J}_{N}(\mathbf{r}, t)-\mathbf{u} \rho_{E^{\prime}}^{\prime}\left(\mathbf{r}^{\prime}, t\right) .
\end{aligned}
$$

The local velocity $\mathbf{u}(\mathbf{r}, t)$ of the fluid at a given point $\mathbf{r}$ is defined by imposing that, in a Galilean frame with uniform velocity $\mathbf{u}=\mathbf{u}(\mathbf{r}, t)$, the density of momentum $\rho_{\mathbf{P}^{\prime}}^{\prime}\left(\mathbf{r}^{\prime}, t\right)$ at the corresponding moving point (or equivalently the current density of particles $\left.\mathbf{J}_{N}^{\prime}\left(\mathbf{r}^{\prime}, t\right)\right)$ vanishes. Introducing the mass density $\rho=m \rho_{N}$, we have

$$
m \mathbf{J}_{N}(\mathbf{r}, t)=\rho_{\mathbf{P}}(\mathbf{r}, t) \equiv \mathbf{u}(\mathbf{r}, t) \rho(\mathbf{r}, t),
$$

that is, using Eqs. (4) and (5),

$$
\mathbf{u}(\mathbf{r}, t)=\frac{\int \mathrm{d}^{3} \mathbf{p} \frac{\mathbf{p}}{m} f(\mathbf{r}, \mathbf{p} ; t)}{\int \mathrm{d}^{3} \mathbf{p} f(\mathbf{r}, \mathbf{p} ; t)} .
$$

The relations (16) and (17) suggest to parametrise $\rho_{E}, \mathbf{J}_{\mathbf{P}}$ and $\mathbf{J}_{E}$ in terms of $\rho(\mathbf{r}, t)$, of $\mathbf{u}(\mathbf{r}, t)$, and of the quantities $\rho_{E^{\prime}}^{\prime} \equiv \rho_{U}, \mathbf{J}_{\mathbf{P}^{\prime}}^{\prime} \equiv \boldsymbol{\sigma}$ and $\mathbf{J}_{E^{\prime}}^{\prime} \equiv \mathbf{J}_{Q}$ which pertain to the local rest frame, according to

$$
\begin{gathered}
\rho_{E}=\frac{1}{2} \rho u^{2}+\rho_{U}, \\
\mathbf{J}_{\mathbf{P} \beta}^{\alpha}=u_{\alpha} u_{\beta} \rho(\mathbf{r}, t)+\boldsymbol{\sigma}_{\beta}^{\alpha}, \\
\mathbf{J}_{E}=\left(\frac{1}{2} \rho u^{2}+\rho_{U}\right) \mathbf{u}+\sum_{\beta} u_{\beta} \sigma_{\beta}^{\alpha}+\mathbf{J}_{Q},
\end{gathered}
$$

all these quantities being functions of $\mathbf{r}$ and $t$. Microscopic expressions for $\rho_{U}, \boldsymbol{\sigma}$ and $\mathbf{J}_{Q}$ are obtained by inserting (8), (10) and (11) into Eqs. (20)-(22). The stress tensor $\boldsymbol{\sigma}$ has then the form (8) where $p_{\alpha} p_{\beta}$ is replaced by $\left(p_{\alpha}-m u_{\alpha}\right)\left(p_{\beta}-m u_{\beta}\right)$, the density of internal energy $\rho_{U}$ has the form $(10)$ where $p^{2}$ is replaced by $(\mathbf{p}-m \mathbf{u})^{2}$, and the heat flux $\mathbf{J}_{Q}$ the form (11) where each $p_{\alpha}$ is replaced by $\left(p_{\alpha}-m u_{\alpha}\right)$ in the factors that precede $f$ and $f_{2}$.

We have interpreted $\frac{1}{2} \rho u^{2}$ as the density of kinetic energy, $\rho_{U}$ as the density of internal energy in the local rest frame, $\boldsymbol{\sigma}$ as the stress tensor and $\mathbf{J}_{Q}$ as the heat flow. These interpretations are confirmed by rewriting the conservation laws in terms of the new quantities. We first get the mass conservation

$$
\frac{\partial \rho}{\partial t}+\operatorname{div} \rho \mathbf{u}=0
$$

Then, the conservation of momentum yields

$$
\rho\left[\frac{\partial u_{\beta}}{\partial t}+\sum_{\alpha} u_{\alpha} \frac{\partial u_{\beta}}{\partial r_{\alpha}}\right]=-\sum_{\alpha} \frac{\partial \boldsymbol{\sigma}_{\beta}^{\alpha}}{\partial r_{\alpha}},
$$

which is identified with Newton's equation in the Eulerian description, applied to an infinitesimal volume element around the point $\mathbf{r}$. The conservation of energy, rewritten as 


$$
\frac{\partial \rho_{U}}{\partial t}+\sum_{\alpha} u_{\alpha} \frac{\partial \rho_{U}}{\partial r_{\alpha}}=-\sum_{\alpha \beta} \boldsymbol{\sigma}_{\beta}^{\alpha} \frac{\partial u_{\beta}}{\partial r_{\alpha}}-\operatorname{div} \mathbf{J}_{Q},
$$

exhibits the balance of work done by the stresses on the volume element and of heat that it receives. The conservation of angular momentum entails the symmetry of the stress tensor $\boldsymbol{\sigma}_{\beta}^{\alpha}$.

These well-known laws have been recovered here in an arbitrary situation, possibly far from local equilibrium. In the hydrodynamic regime, they would be complemented by Fourier's heat law and by the equations of viscosity, but in the general case considered here the local temperature and the local chemical potential are not defined. We can still rely, however, on the dynamical laws (23)-(25) that relate the various quantities expressed by the above microscopic formulae.

\section{References}

[1] J. Yvon, La théorie statistique des fluides et l'équation d'état, Actual. Sci. et Indust. $\mathrm{N}^{\circ}$ 203 (Hermann, Paris, 1935).

[2] R.Balian, From microphysics to macrophysics, Vol. II, Chap. 14 (Springer, 2007). 